\title{
ANALYSIS OF TRIANGULAR TOWER ON BUILDING SUBJECTED TO SEISMIC LOADS WITH DIFFERENT POSITIONS
}

\author{
Siddharth Pastariya \\ Department of Civil Engineering \\ Sri Aurobindo Institute of Technology Indore, M.P, India
}

\begin{abstract}
In urban areas, the scarcity of land is a serious issue now a days with increasing the population count. It is well known fact that if the population count increases, the networking between them is also increases. Since the area of lant is less for telecommunication tower, the companies are now installing it on the roof of the multistory building to continue their supply. In this work, it has shown that the response of the triangular tower located at $\mathbf{G + 6}$ and $\mathbf{G + 1 0}$ storied building. Total 10 models were taken and studied for different parameters and most efficient model is selected which has least parametric values among all by the help of Staad pro.
\end{abstract}

Keywords: Rooftop Telecommunication Tower, Square base, Seismic Effects, Response spectrum, Staad pro.

\section{INTRODUCTION}

Telecommunication towers made up of steel angle sections used to fix the anteena along with all the fixtures used in mobile communication at an optimum location and bear the load of the same and transfer it to the ground. The members of the tower transfer the loads from its intermediate members. The main approach is to transfer the total load of the telecommunication tower to the roof of the multistoried structure. The structure then transfer the upcoming load from beam column arrangement to the foundation. The main criteria in this approach is the position of tower on the roof such that the structure will not get much effect from the load of telecommunication tower. If the extra load bearing members was provided in the structure to transfer the load, it was provided only to support the self load of the structure. The position of tower will affect if there was not any of this structural member present beneath. The tower bracings are also affect the structure such that load coming from the tower might be different. $\mathrm{X}$ bracing and Chevron type bracings are the most common one used in telecommunication tower.

\section{OBJECTIVE}

This study examines different parameters like displacement, moments, stresses, shear and axial forces. The telecommunication tower is placed on roof of a building at different locations, at medium soil condition under seismic forces for earthquake zone IV.

\section{STRUCTURE MODELING}

The space frame has been modeled in Staad pro software. The descriptions of the host structure and tower are listed in Table 1 and details of loading used in this work listed in Table 2. The connections assumed to be rigid at base for host structure and also for triangular base tower. The arrangement idealization of triangular base tower is shown in Figure 1; Figure 2 shows different locations of triangular tower on roof respectively to show the various parametric analysis.

Table 1: Details of building \& triangular base rooftop tower

\begin{tabular}{|c|c|}
\hline Building configuration & $G+6 \& G+10$ \\
\hline Height of building & $28.62 \mathrm{~m}$ and $43.26 \mathrm{~m}$ \\
\hline Dimensions of building & $15 \mathrm{~m} \times 9 \mathrm{~m}$ \\
\hline Size of beam & $400 \mathrm{~mm} \times 300 \mathrm{~mm}$ \\
\hline Size of column & $450 \mathrm{~mm} \times 450 \mathrm{~mm}$ \\
\hline $\begin{array}{l}\text { Concrete and Steel } \\
\text { Grade }\end{array}$ & $\begin{array}{l}\text { M25 \& Fe } 415 \\
\text { grade }\end{array}$ \\
\hline $\begin{array}{l}\text { Height of tower (square } \\
\text { base) }\end{array}$ & $15 \mathrm{~m}$ \\
\hline $\begin{array}{l}\text { Effective base width } \\
\text { (square base) }\end{array}$ & $3 \mathrm{~m}$ wide \\
\hline $\begin{array}{l}\text { Panel height (square } \\
\text { base) }\end{array}$ & $1.5 \mathrm{~m}$ long \\
\hline $\begin{array}{l}\text { Horizontal and vertical } \\
\text { steel members }\end{array}$ & ISA $110 \times 110 \times 8$ \\
\hline Inclined members & ISA $90 \times 90 \times 12$ \\
\hline
\end{tabular}


International Journal of Engineering Applied Sciences and Technology, 2020

Vol. 4, Issue 12, ISSN No. 2455-2143, Pages 642-650

Published Online April 2020 in IJEAST (http://www.ijeast.com)

Table 2: Details of loading

\begin{tabular}{|l|l|}
\hline Earthquake parameters & $\begin{array}{l}\text { Zone IV with RF 5 \& } \\
5 \% \text { damping ratio }\end{array}$ \\
\hline Period in X \& Z direction & $\begin{array}{l}1.2978 \text { \& } 1.0052 \\
\text { seconds }\end{array}$ \\
\hline $\begin{array}{l}\text { Dead load for floor and } \\
\text { roof }\end{array}$ & $\begin{array}{l}12 \mathrm{KN} / \mathrm{m}^{2} \& 10 \\
\mathrm{KN} / \mathrm{m}^{2}\end{array}$ \\
\hline $\begin{array}{l}\text { Live load for floor and } \\
\text { roof }\end{array}$ & $4 \mathrm{KN} / \mathrm{m}^{2} \& 2 \mathrm{KN} / \mathrm{m}^{2}$ \\
\hline
\end{tabular}

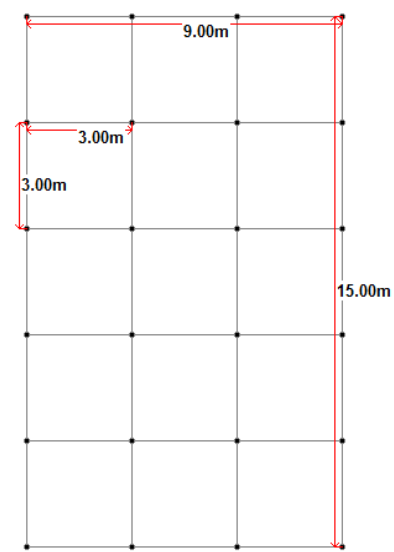

Figure 1: Plan Model -1 \& 6

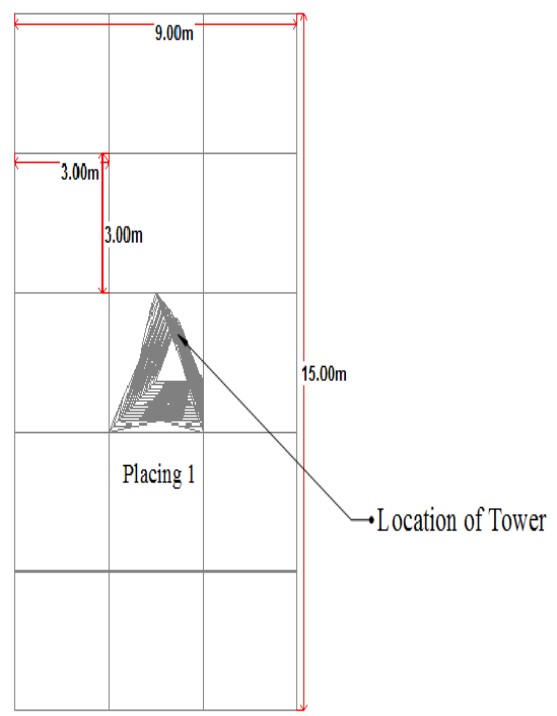

Figure 2: Plan Model -2 \& 7

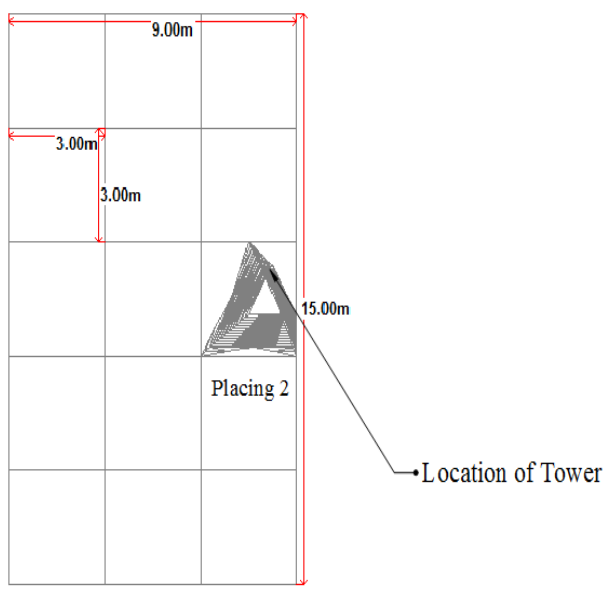

Figure 3: Plan Model -3 \& 8

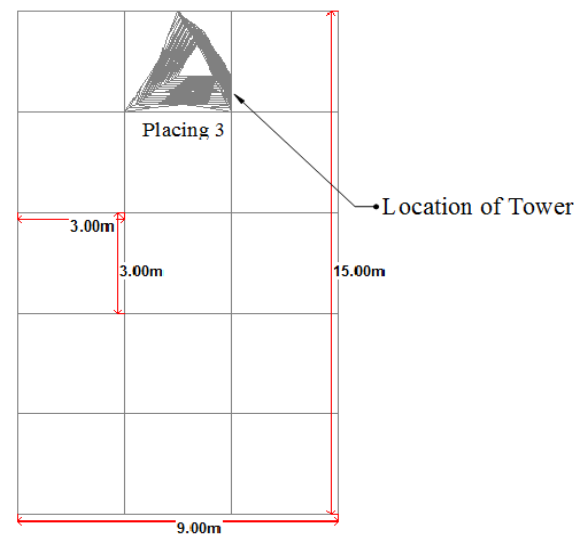

Figure 4: Plan Model -4 \& 9

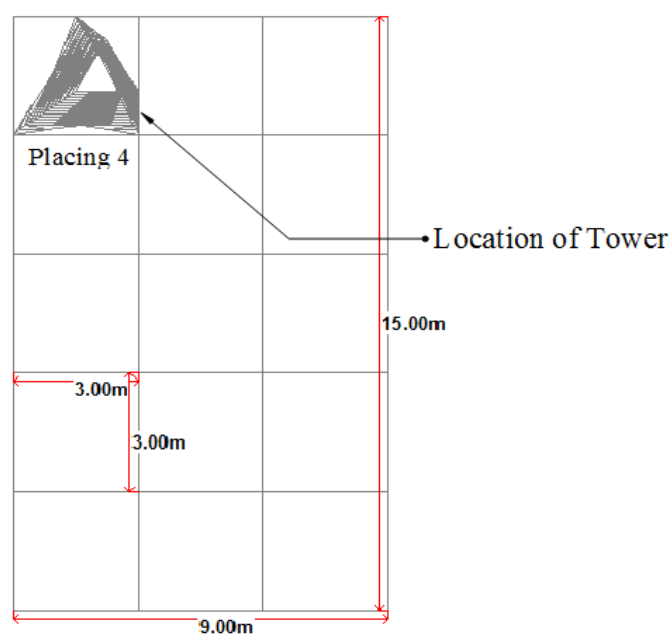

Figure 5: Plan Model -5 \& 10

\section{RESULTS AND DISCUSSION}

As per the objectives, the Response Spectrum Analysis has been performed on different models consist of placing without tower $(\mathrm{G}+6)$ model, placing with tower $(\mathrm{G}+6)$ all models, placing without tower $(\mathrm{G}+10)$ model and placing with 
tower $(\mathrm{G}+10)$ all models for different location of telecommunication tower on roof are assessed for building areas which is situated in earthquake zones i.e. Zone IV.

The analysis results obtained using Staad pro software is shown in tabular form along with various graphs with various parameters as follows:

Table 3: Shear Forces and Moments in Building (Beam Parallel to X direction) for different Models

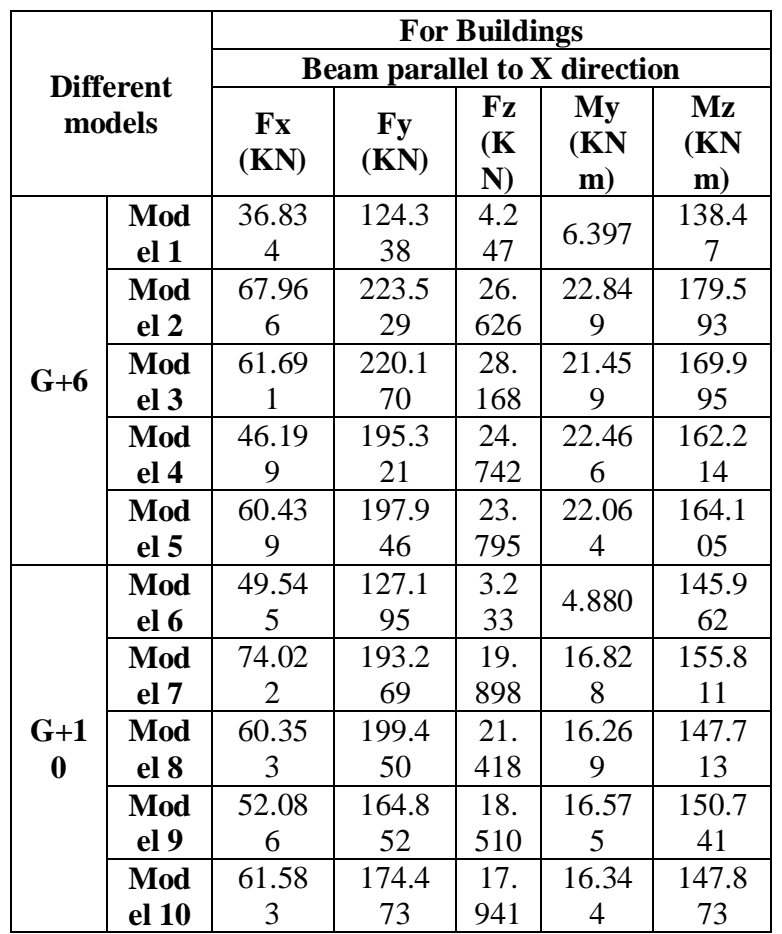

Minimum value of shear forces and moment when beams are parallel to $\mathrm{X}$ direction seems to be in model 4 in G+6 storey building and model 9 in $\mathrm{G}+10$ storey building. Hence by observing this least values, model 4 and 9 should be preferred.

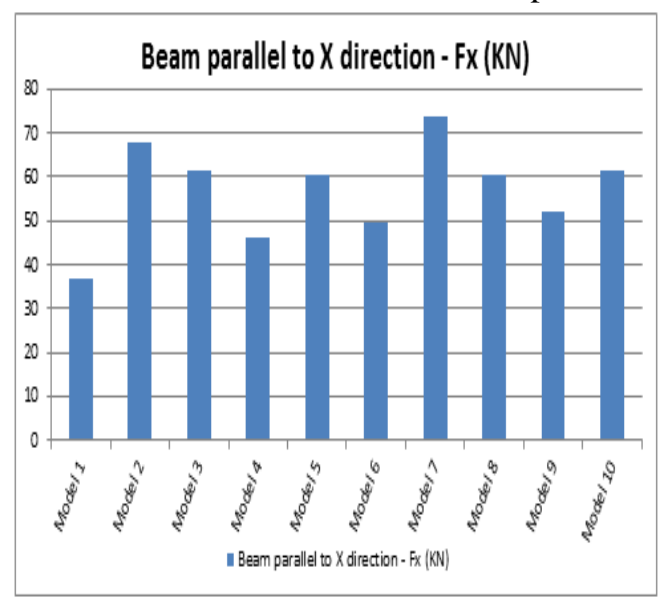

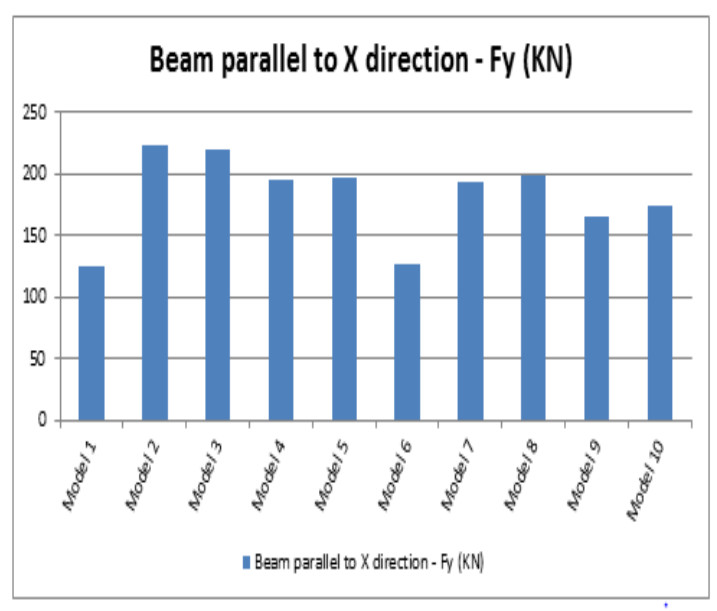

Graph 1: Graphical representation of Shear Forces (Fx Graph 2: Graphical representation of Shear Forces (Fy)
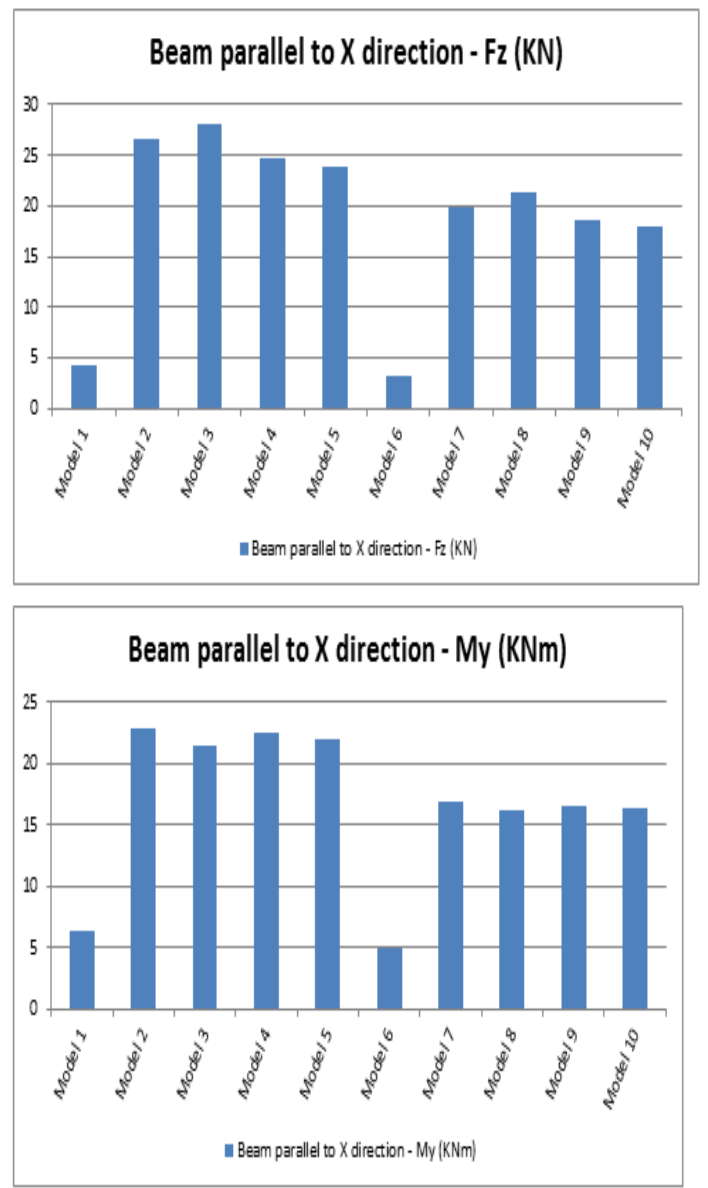

Graph 3: Graphical representation of Shear Forces (Fz) Graph 4: Graphical representation of Moment (My) 


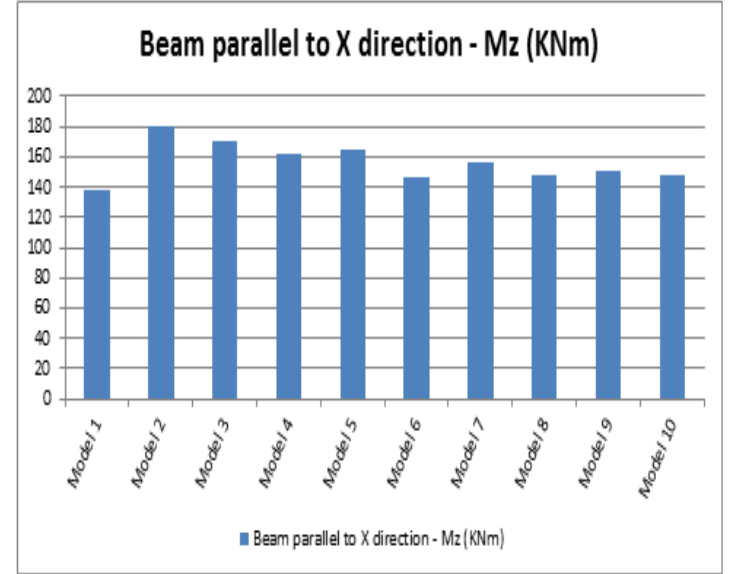

Graph 5: Graphical representation of Moments (Mz)

Table 4: Shear Forces and Moments in Building (Beam Parallel to Y direction) for different Models

\begin{tabular}{|c|c|c|c|c|c|c|}
\hline & \multirow{2}{*}{\multicolumn{5}{|c|}{$\begin{array}{c}\text { For Buildings } \\
\text { Beam parallel to Y direction }\end{array}$}} \\
\hline & & & & & & \\
\hline & & $\begin{array}{c}\mathbf{F x} \\
(\mathbf{K N} \\
)\end{array}$ & $\begin{array}{c}\mathbf{F y} \\
(\mathbf{K N} \\
)\end{array}$ & \multirow{2}{*}{$\begin{array}{c}\mathbf{F z} \\
(\mathbf{K N} \\
) \\
62.1 \\
34\end{array}$} & \multirow{2}{*}{$\begin{array}{c}\begin{array}{c}\text { My } \\
(\mathbf{K N} \\
\mathbf{m})\end{array} \\
117 . \\
158\end{array}$} & \multirow{2}{*}{$\begin{array}{c}\begin{array}{c}\mathbf{M z} \\
(\mathbf{K N} \\
\mathbf{m})\end{array} \\
99.6 \\
01\end{array}$} \\
\hline \multirow{5}{*}{$\begin{array}{c}\text { G+ } \\
6\end{array}$} & $\begin{array}{l}\text { Mode } \\
11\end{array}$ & $\begin{array}{l}1930 \\
.154\end{array}$ & $\begin{array}{c}53.6 \\
71\end{array}$ & & & \\
\hline & $\begin{array}{l}\text { Mode } \\
12\end{array}$ & $\begin{array}{l}2043 \\
.435\end{array}$ & $\begin{array}{c}55.0 \\
01\end{array}$ & $\begin{array}{c}64.2 \\
97\end{array}$ & $\begin{array}{l}120 . \\
067\end{array}$ & $\begin{array}{l}105 . \\
143\end{array}$ \\
\hline & $\begin{array}{l}\text { Mode } \\
13\end{array}$ & $\begin{array}{l}2008 \\
.606\end{array}$ & $\begin{array}{c}55.3 \\
09\end{array}$ & $\begin{array}{c}64.2 \\
32\end{array}$ & $\begin{array}{l}120 . \\
620\end{array}$ & $\begin{array}{l}124 . \\
323\end{array}$ \\
\hline & Mode & $\begin{array}{l}1952 \\
.751\end{array}$ & $\begin{array}{c}55.8 \\
48\end{array}$ & $\begin{array}{c}62.9 \\
71\end{array}$ & $\begin{array}{l}118 . \\
781\end{array}$ & 104. \\
\hline & $\begin{array}{l}\text { Mode } \\
15\end{array}$ & $\begin{array}{l}1953 \\
.293\end{array}$ & $\begin{array}{c}56.5 \\
81\end{array}$ & $\begin{array}{c}63.2 \\
65\end{array}$ & $\begin{array}{l}119 . \\
351\end{array}$ & $\begin{array}{l}113 . \\
938\end{array}$ \\
\hline \multirow{6}{*}{$\begin{array}{l}\text { G+ } \\
10\end{array}$} & $\begin{array}{c}\text { Mode } \\
16\end{array}$ & $\begin{array}{r}2699 \\
.864\end{array}$ & $\begin{array}{c}55.4 \\
39\end{array}$ & $\begin{array}{c}63.4 \\
41\end{array}$ & $\begin{array}{l}116 . \\
689\end{array}$ & $\begin{array}{l}101 . \\
517\end{array}$ \\
\hline & $\begin{array}{l}\text { Mode } \\
17\end{array}$ & $\begin{array}{c}2776 \\
.011\end{array}$ & $\begin{array}{c}56.6 \\
66\end{array}$ & $\begin{array}{c}65.3 \\
5\end{array}$ & $\begin{array}{l}120 . \\
235\end{array}$ & $\begin{array}{l}106 . \\
038\end{array}$ \\
\hline & Mode & 2764 & 56.6 & 65.2 & 120. & 122. \\
\hline & 18 & .260 & 6 & 79 & 087 & 485 \\
\hline & $\begin{array}{c}\text { Mode } \\
19\end{array}$ & $\begin{array}{c}2728 \\
.62\end{array}$ & $\begin{array}{c}56.8 \\
78\end{array}$ & $\begin{array}{c}64.0 \\
88\end{array}$ & $\begin{array}{l}117 . \\
874\end{array}$ & $\begin{array}{l}104 . \\
123\end{array}$ \\
\hline & $\begin{array}{c}\text { Mode } \\
1 \text { 10 }\end{array}$ & $\begin{array}{c}2730 \\
.72\end{array}$ & $\begin{array}{c}57.2 \\
38\end{array}$ & $\begin{array}{c}64.2 \\
86\end{array}$ & $\begin{array}{l}118 . \\
234\end{array}$ & $\begin{array}{l}107 . \\
365\end{array}$ \\
\hline
\end{tabular}

Minimum value of shear forces and moment when beams are parallel to $\mathrm{Y}$ direction seems to be in model 4 in G+6 storey building and model 9 in $\mathrm{G}+10$ storey building. Hence by observing this least values, model 4 and 9 should be preferred.
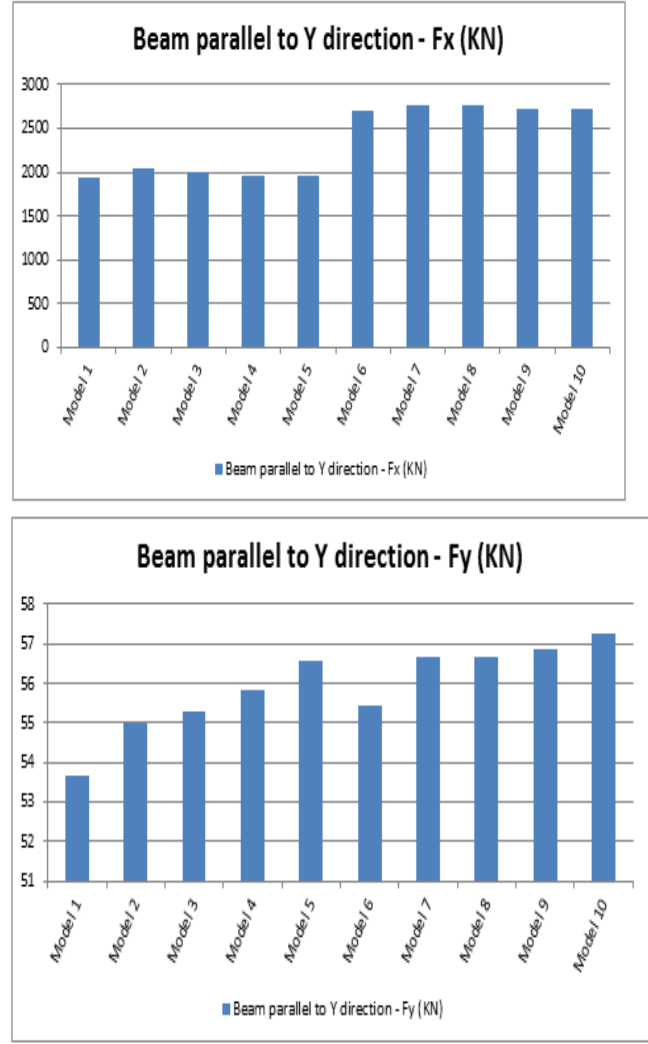

Graph 6: Graphical representation of Shear Forces (Fx) Graph 7: Graphical representation of Shear Force (Fy)
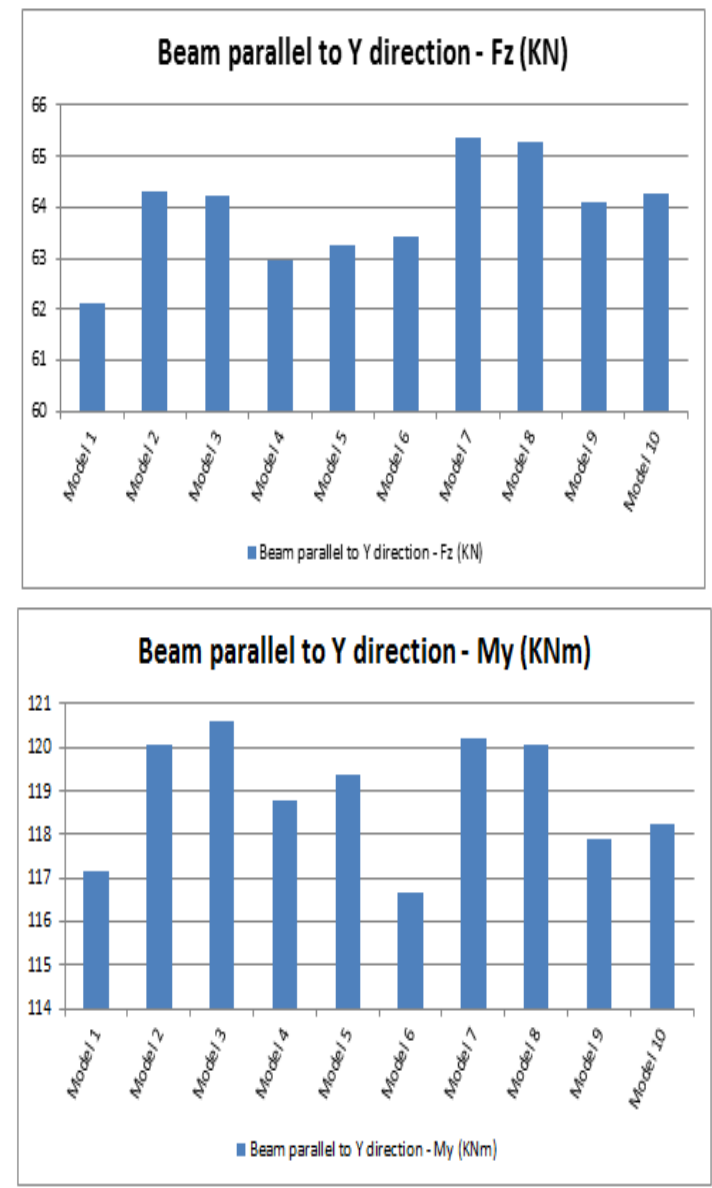

Graph 8: Graphical representation of Shear Forces (Fz) Graph 9: Graphical representation of Moments (My) 


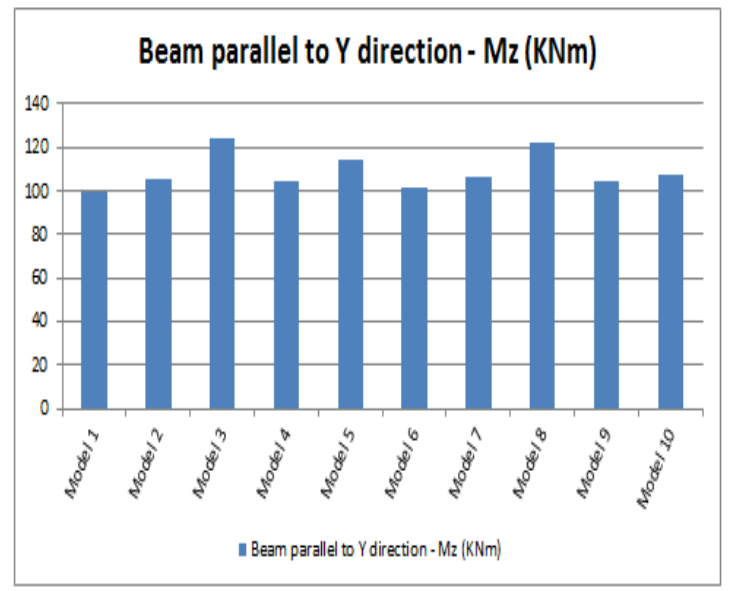

Graph 10: Graphical representation of Moments (Mz)

Table 5: Shear Forces and Moments in Building (Beam Parallel to $\mathrm{Z}$ direction) for different Models

\begin{tabular}{|c|c|c|c|c|c|c|}
\hline \multirow{3}{*}{\multicolumn{2}{|c|}{$\begin{array}{c}\text { Different } \\
\text { models }\end{array}$}} & \multirow{2}{*}{\multicolumn{5}{|c|}{$\begin{array}{c}\text { For Buildings } \\
\text { Beam parallel to } \mathrm{Z} \text { direction }\end{array}$}} \\
\hline & & & & & & \\
\hline & & \multirow{2}{*}{$\begin{array}{c}\mathbf{F x} \\
(\mathbf{K N} \\
) \\
36.0 \\
78\end{array}$} & \multirow{2}{*}{$\begin{array}{c}\begin{array}{c}\mathbf{F y} \\
(\mathbf{K N})\end{array} \\
\begin{array}{c}127.6 \\
37\end{array}\end{array}$} & \multirow{2}{*}{$\begin{array}{l}\mathbf{F z} \\
(\mathbf{K} \\
\mathbf{N}) \\
4.1 \\
15\end{array}$} & \multirow{2}{*}{$\begin{array}{c}\begin{array}{c}\text { My } \\
(\mathbf{K N}\end{array} \\
\mathbf{m})\end{array}$} & \multirow{2}{*}{$\begin{array}{c}\begin{array}{c}\mathbf{M z} \\
(\mathbf{K N}\end{array} \\
\mathbf{m})\end{array}$} \\
\hline \multirow{5}{*}{$\begin{array}{c}\text { G+ } \\
6\end{array}$} & $\begin{array}{c}\text { Mo } \\
\text { del } \\
1\end{array}$ & & & & & \\
\hline & $\begin{array}{c}\text { Mo } \\
\text { del } \\
2\end{array}$ & $\begin{array}{c}45.4 \\
47\end{array}$ & $\begin{array}{c}131.2 \\
81\end{array}$ & $\begin{array}{l}7.3 \\
27\end{array}$ & $\begin{array}{c}12.4 \\
02\end{array}$ & $\begin{array}{c}155.7 \\
84\end{array}$ \\
\hline & $\begin{array}{c}\text { Mo } \\
\text { del } \\
\mathbf{3}\end{array}$ & $\begin{array}{c}43.1 \\
66\end{array}$ & $\begin{array}{c}129.5 \\
70\end{array}$ & $\begin{array}{l}6.5 \\
96\end{array}$ & $\begin{array}{c}11.5 \\
95\end{array}$ & $\begin{array}{c}156.0 \\
17\end{array}$ \\
\hline & $\begin{array}{c}\text { Mo } \\
\text { del } \\
4\end{array}$ & $\begin{array}{c}65.8 \\
81\end{array}$ & $\begin{array}{c}128.6 \\
42\end{array}$ & $\begin{array}{l}8.6 \\
63\end{array}$ & $\begin{array}{c}15.0 \\
24\end{array}$ & $\begin{array}{c}154.4 \\
38\end{array}$ \\
\hline & $\begin{array}{c}\text { Mo } \\
\text { del } \\
5\end{array}$ & $\begin{array}{c}65.9 \\
53\end{array}$ & $\begin{array}{c}129.0 \\
02\end{array}$ & $\begin{array}{l}9.2 \\
43\end{array}$ & $\begin{array}{c}14.3 \\
57\end{array}$ & $\begin{array}{c}155.0 \\
04\end{array}$ \\
\hline \multirow{5}{*}{$\begin{array}{l}\text { G+ } \\
10\end{array}$} & $\begin{array}{c}\text { Mo } \\
\text { del } \\
6\end{array}$ & $\begin{array}{c}53.3 \\
11\end{array}$ & $\begin{array}{c}129.9 \\
73\end{array}$ & $\begin{array}{l}3.0 \\
90\end{array}$ & $\begin{array}{c}4.79 \\
9\end{array}$ & $\begin{array}{c}156.5 \\
57\end{array}$ \\
\hline & $\begin{array}{c}\text { Mo } \\
\text { del } \\
7\end{array}$ & $\begin{array}{c}57.5 \\
58\end{array}$ & $\begin{array}{c}131.5 \\
37\end{array}$ & $\begin{array}{l}5.5 \\
26\end{array}$ & $\begin{array}{c}9.32 \\
4\end{array}$ & $\begin{array}{c}159.0 \\
99\end{array}$ \\
\hline & $\begin{array}{c}\text { Mo } \\
\text { del } \\
8\end{array}$ & $\begin{array}{c}55.9 \\
25\end{array}$ & $\begin{array}{c}131.6 \\
12\end{array}$ & $\begin{array}{l}5.2 \\
42\end{array}$ & $\begin{array}{c}9.10 \\
2\end{array}$ & $\begin{array}{c}159.1 \\
87\end{array}$ \\
\hline & $\begin{array}{c}\text { Mo } \\
\text { del } \\
9\end{array}$ & $\begin{array}{c}71.1 \\
24\end{array}$ & $\begin{array}{c}130.5 \\
97\end{array}$ & $\begin{array}{l}6.6 \\
98\end{array}$ & $\begin{array}{c}11.2 \\
54\end{array}$ & $\begin{array}{c}157.5 \\
99\end{array}$ \\
\hline & $\begin{array}{l}\text { Mo } \\
\text { del } \\
10\end{array}$ & $\begin{array}{c}70.8 \\
85\end{array}$ & $\begin{array}{c}130.8 \\
20\end{array}$ & $\begin{array}{l}7.3 \\
90\end{array}$ & $\begin{array}{c}11.6 \\
97\end{array}$ & $\begin{array}{c}157.9 \\
11\end{array}$ \\
\hline
\end{tabular}

Minimum value of shear forces and moment when beams are parallel to $\mathrm{Z}$ direction seems to be in model 4 in G+6 storey building and model 9 in $\mathrm{G}+10$ storey building. Hence by observing this least values, model 4 and 9 should be preferred.
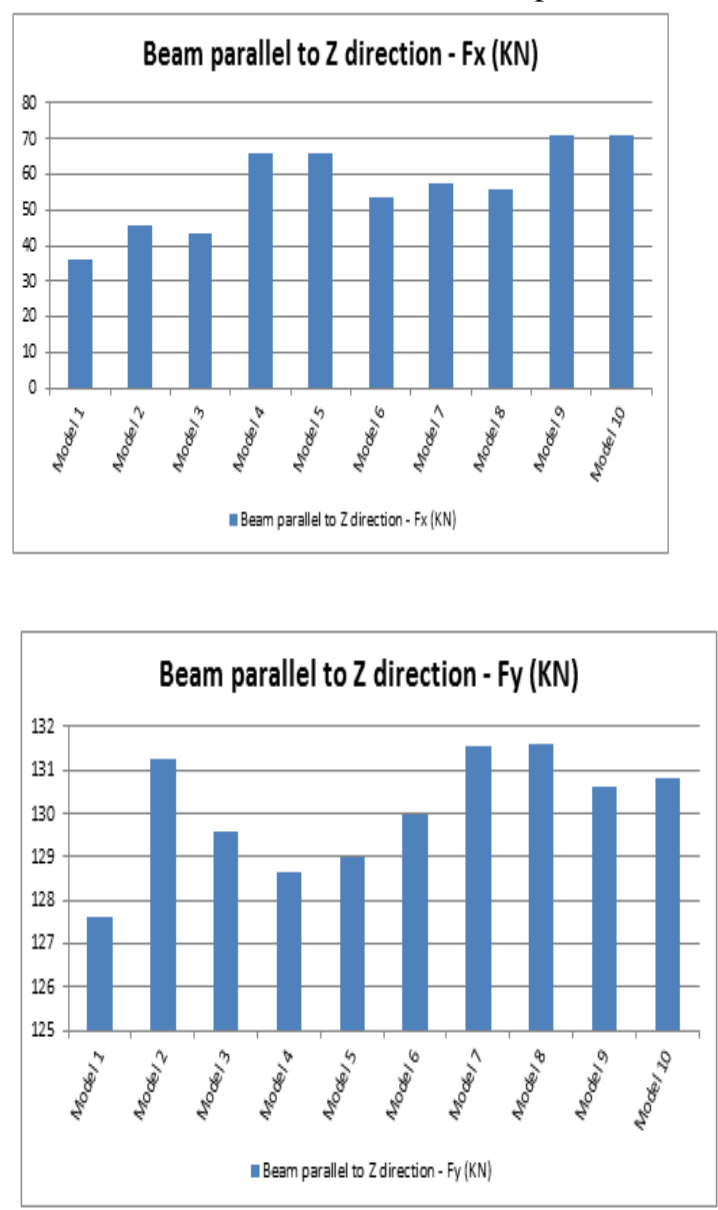

Graph 11: Graphical representation of Shear Forces (Fx) in Building (Beam Parallel to $\mathrm{Z}$ direction) for different Models

Graph 12: Graphical representation of Shear Forcess (Fy) in Building (Beam Parallel to $\mathrm{Z}$ direction) for different Models

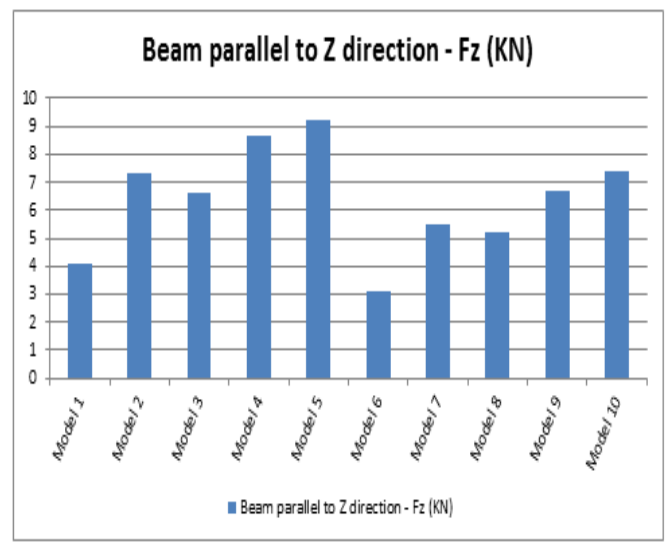




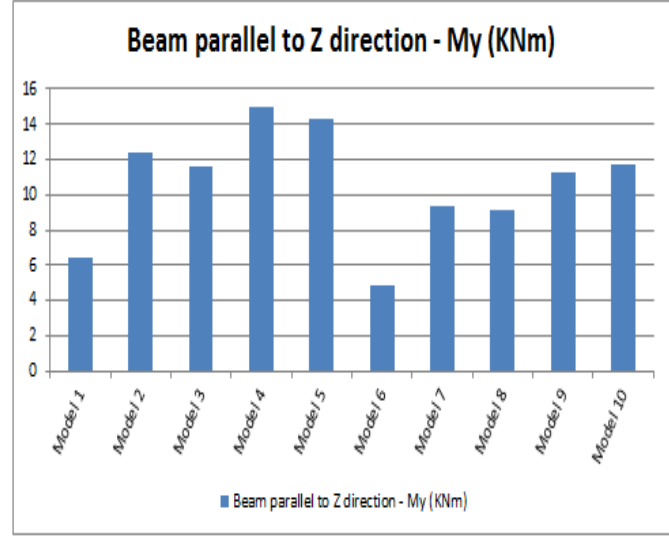

Graph 13: Graphical representation of Shear Forces (Fy) Graph 14: Graphical representation of Moments (My)

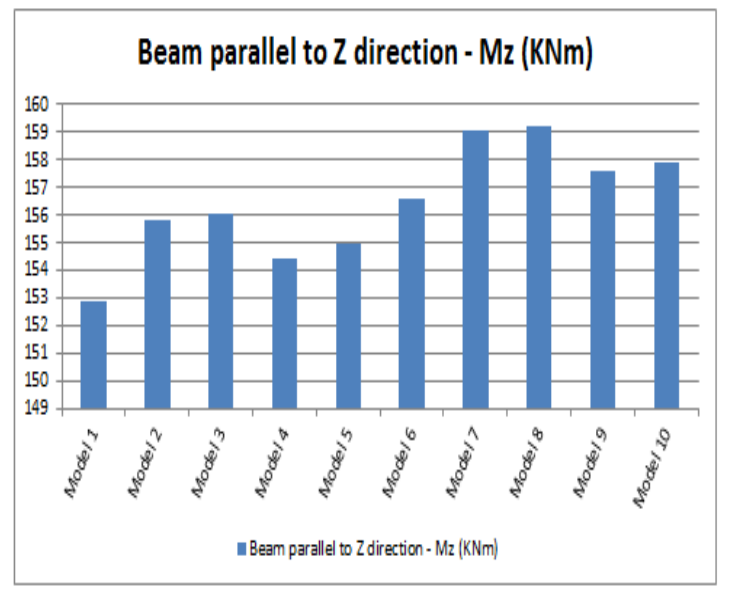

Graph 15: Graphical representation of Moments (Mz)

Table 6: Nodal displacement in Building ( $\mathrm{X}$ and $\mathrm{Z}$ direction) for different Models

\begin{tabular}{|c|c|c|c|}
\hline \multicolumn{2}{|c|}{ Different models } & \multicolumn{2}{c|}{$\begin{array}{c}\text { For Buildings } \\
\text { different directions }\end{array}$} \\
\cline { 3 - 4 } & $\mathbf{X}$ (mm) & Z (mm) \\
\hline \multirow{7}{*}{ G+6 } & $\begin{array}{c}\text { Model } \\
\mathbf{1}\end{array}$ & 48.541 & 55.522 \\
\cline { 2 - 4 } & $\begin{array}{c}\text { Model } \\
\mathbf{2}\end{array}$ & 50.890 & 57.965 \\
\cline { 2 - 4 } & $\begin{array}{c}\text { Model } \\
\mathbf{3}\end{array}$ & 51.379 & 58.287 \\
\cline { 2 - 4 } & $\begin{array}{c}\text { Model } \\
\mathbf{4}\end{array}$ & 51.895 & 58.341 \\
\cline { 2 - 4 } & $\begin{array}{c}\text { Model } \\
\mathbf{5}\end{array}$ & 52.653 & 58.804 \\
\hline \multirow{4}{*}{ G+10 } & $\begin{array}{c}\text { Model } \\
\mathbf{6}\end{array}$ & 81.399 & 89.027 \\
\cline { 2 - 4 } & $\begin{array}{c}\text { Model } \\
\mathbf{7}\end{array}$ & 83.878 & 91.363 \\
\cline { 2 - 4 } & $\begin{array}{c}\text { Model } \\
\mathbf{8}\end{array}$ & 85.033 & 91.772 \\
\hline
\end{tabular}

\begin{tabular}{|l|c|c|c|}
\hline \multirow{2}{*}{} & $\begin{array}{c}\text { Model } \\
\mathbf{9}\end{array}$ & 85.258 & 92.295 \\
\cline { 2 - 4 } & $\begin{array}{c}\text { Model } \\
\mathbf{1 0}\end{array}$ & 86.790 & 92.737 \\
\hline
\end{tabular}

Minimum value of nodal displacement seems to be in model 2 for both $\mathrm{X}$ and $\mathrm{Z}$ direction in $\mathrm{G}+6$ storey building and model 7 in $\mathrm{G}+10$ storey building. Hence by observing this least values, model 2 and 7 should be preferred.
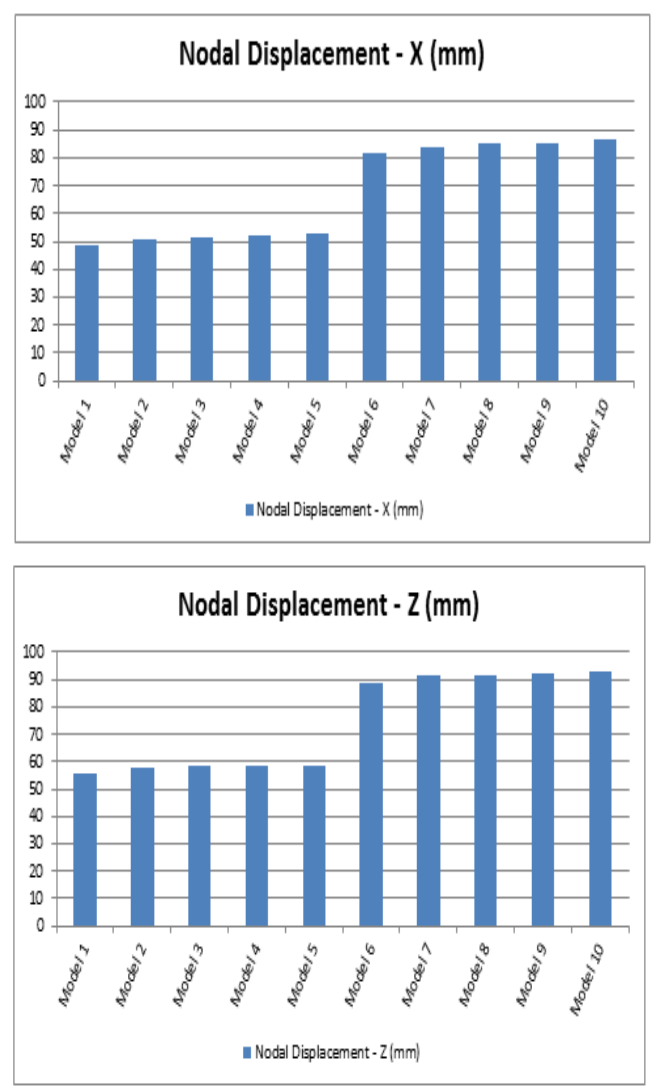

Graph 17: Nodal Displacement in Building (in Z-direction) Graph 16: Nodal Displacement in Building (in X-direction)

Table 7: Storey Drift in Building ( $\mathrm{X}$ and $\mathrm{Z}$ direction) for different Models

\begin{tabular}{|c|c|c|c|}
\hline \multirow{2}{*}{ Different models } & \multicolumn{2}{c|}{ For Buildings } \\
\cline { 3 - 4 } & & \multicolumn{2}{c|}{ Storey Drift } \\
\cline { 3 - 4 } & Model 1 & 0.2031 & $\mathbf{Z}(\mathbf{c m})$ \\
\hline \multirow{4}{*}{ G+6 } & Model 2 & 0.2261 & 0.2384 \\
\cline { 2 - 4 } & Model 3 & 0.2268 & 0.2375 \\
\cline { 2 - 4 } & Model 4 & 0.2286 & 0.2398 \\
\cline { 2 - 4 } & Model 5 & 0.2316 & 0.2410 \\
\hline \multirow{4}{*}{ G+10 } & Model 6 & 0.1728 & 0.2340 \\
\cline { 2 - 4 } & Model 7 & 0.1649 & 0.2250 \\
\cline { 2 - 4 } & Model 8 & 0.2446 & 0.2227 \\
\cline { 2 - 4 } & Model 9 & 0.2486 & 0.2245 \\
\cline { 2 - 4 } & Model 10 & 0.2507 & 0.2258 \\
\hline
\end{tabular}


Minimum value of storey drift seems to be in model 2 for both $\mathrm{X}$ and $\mathrm{Z}$ direction in $\mathrm{G}+6$ storey building and model 7 in G+10 storey building. Hence by observing this least values, model 2 and 7 should be preferred.

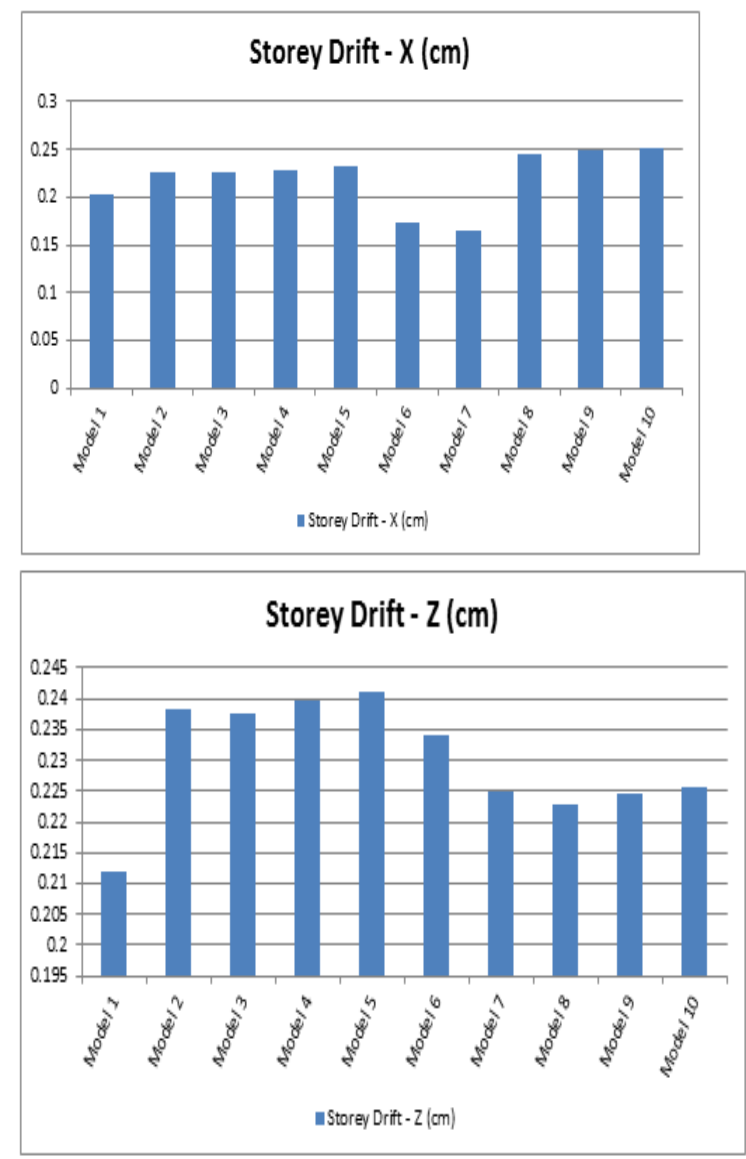

Graph 18: Storey Drift in Building (in X-direction) Graph 19: Storey Drift in Building (in Z-direction)

Table 8: Nodal displacement ( $\mathrm{X}$ and $\mathrm{Z}$ direction) and Axial Forces (Compressive and Tensile) in Tower for different Models

\begin{tabular}{|c|c|c|c|c|c|}
\hline \multirow{3}{*}{\multicolumn{2}{|c|}{$\begin{array}{c}\text { Different } \\
\text { models }\end{array}$}} & \multicolumn{4}{|c|}{ For Towers } \\
\hline & & \multicolumn{2}{|c|}{$\begin{array}{c}\text { Nodal } \\
\text { Displacement }\end{array}$} & \multicolumn{2}{|c|}{ Axial Force } \\
\hline & & $\mathbf{X}$ & $\mathbf{Z}$ & Compres & Tensi \\
\hline \multirow{5}{*}{$\begin{array}{c}\text { G+ } \\
6\end{array}$} & $\begin{array}{c}\text { Mod } \\
\text { el } 1\end{array}$ & - & - & - & - \\
\hline & $\begin{array}{c}\text { Mod } \\
\text { el } 2\end{array}$ & $\begin{array}{c}76.89 \\
3\end{array}$ & $\begin{array}{c}89.34 \\
5\end{array}$ & 265.449 & $\begin{array}{c}265.1 \\
51\end{array}$ \\
\hline & $\begin{array}{c}\text { Mod } \\
\text { el 3 }\end{array}$ & $\begin{array}{c}76.73 \\
4\end{array}$ & $\begin{array}{c}89.63 \\
3\end{array}$ & 265.673 & $\begin{array}{c}265.3 \\
75\end{array}$ \\
\hline & $\begin{array}{c}\text { Mod } \\
\text { el } 4\end{array}$ & $\begin{array}{c}74.58 \\
2\end{array}$ & $\begin{array}{c}88.34 \\
1\end{array}$ & 264.932 & $\begin{array}{c}326.6 \\
34\end{array}$ \\
\hline & $\begin{array}{c}\text { Mod } \\
\text { el } 5\end{array}$ & $\begin{array}{c}89.56 \\
5\end{array}$ & $\begin{array}{c}88.08 \\
9\end{array}$ & 265.098 & $\begin{array}{c}264.8 \\
00\end{array}$ \\
\hline G+ & Mod & - & - & - & - \\
\hline
\end{tabular}

\begin{tabular}{|c|c|c|c|c|c|}
\hline 10 & el 6 & & & & \\
\cline { 2 - 6 } & $\begin{array}{c}\text { Mod } \\
\text { el 7 }\end{array}$ & $\begin{array}{c}104.8 \\
93\end{array}$ & $\begin{array}{c}116.3 \\
45\end{array}$ & 265.461 & $\begin{array}{c}225.6 \\
61\end{array}$ \\
\cline { 2 - 6 } & $\begin{array}{c}\text { Mod } \\
\text { el 8 }\end{array}$ & $\begin{array}{c}108.8 \\
42\end{array}$ & $\begin{array}{c}115.6 \\
87\end{array}$ & 265.679 & $\begin{array}{c}225.6 \\
27\end{array}$ \\
\cline { 2 - 6 } & $\begin{array}{c}\text { Mod } \\
\text { el 9 }\end{array}$ & $\begin{array}{c}106.8 \\
57\end{array}$ & $\begin{array}{c}115.6 \\
00\end{array}$ & 264.943 & $\begin{array}{c}224.6 \\
79\end{array}$ \\
\cline { 2 - 6 } & $\begin{array}{c}\text { Mod } \\
\text { el 10 }\end{array}$ & $\begin{array}{c}123.6 \\
19\end{array}$ & $\begin{array}{c}114.0 \\
89\end{array}$ & 265.104 & $\begin{array}{c}224.8 \\
66\end{array}$ \\
\hline
\end{tabular}

Minimum value of nodal displacement and axial forces in steel tower seems to be in model 4 and 9 for both $\mathrm{X}$ and $\mathrm{Z}$ direction in $\mathrm{G}+6$ and in $\mathrm{G}+10$ storey building. Hence by observing this least values, model 4 and 9 should be preferred.
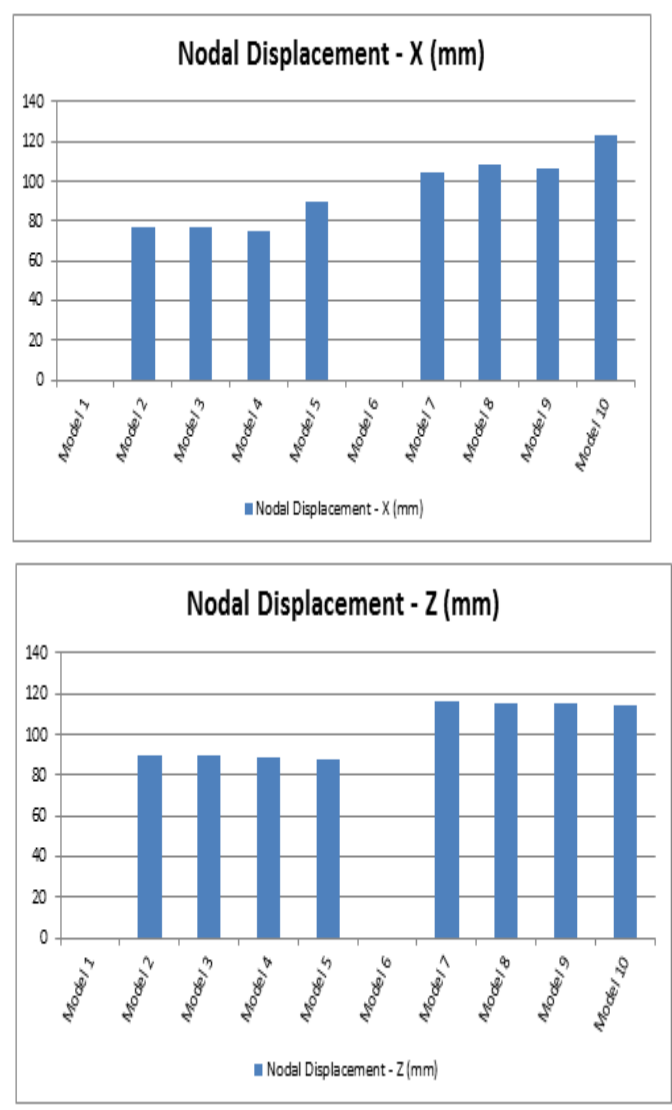

Graph 20: Nodal Displacement in Tower

Graph 21: Nodal Displacement in Tower

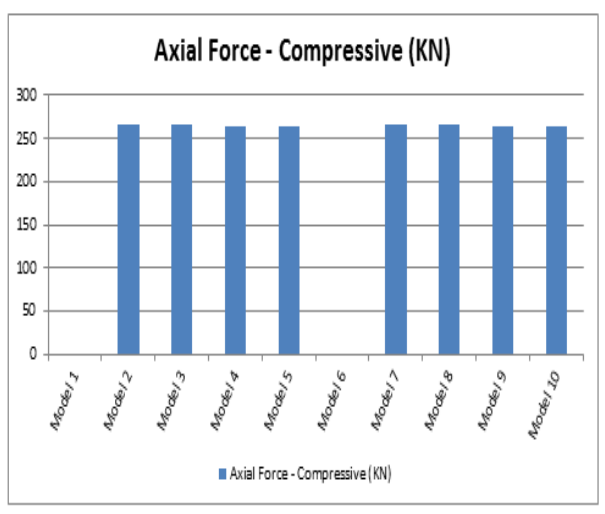




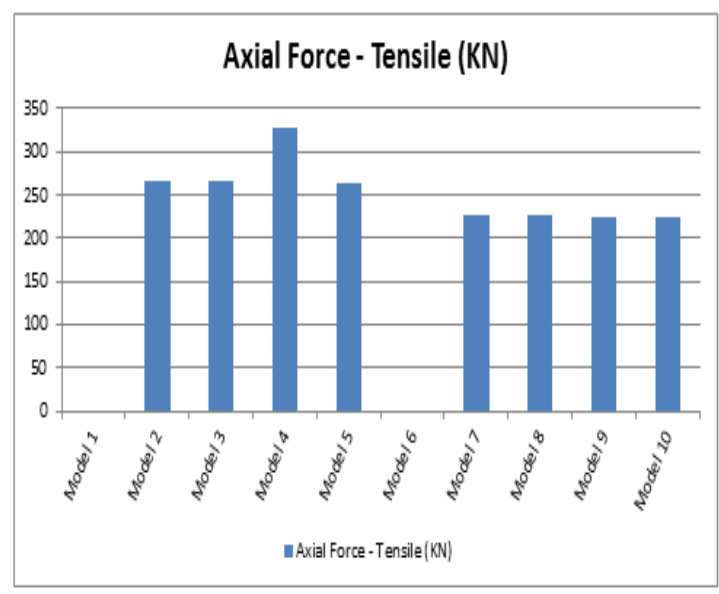

Graph 22: Axial Forces in Tower (Compressive) Graph 23: Axial Forces in Tower(Tensile)

\section{CONCLUSIONS}

1. It is found that when determining shear forces $\mathrm{Fx}, \mathrm{Fy}$ and Fz for building when beam parallel to $\mathrm{X}$ direction, other than model 1 and 6 which is structure without tower, the value of Fx, Fy and $\mathrm{Fz}$ has seems to be least in model 4 and model 9. When determining moments My and $\mathrm{Mz}$ when beam parallel to $\mathrm{X}$ direction, other than model 1 and 6 which is structure without tower, model 3 and model 8 expresses the least values.

2. For building when obtaining shear forces Fx, $\mathrm{Fy}$ and $\mathrm{Fz}$ when beam parallel to $\mathrm{Y}$ direction, other than model without tower, the value of Fx, Fy and Fz has seems to be least in model 4 and model 9. When determining moments My and $\mathrm{Mz}$ when beam parallel to $\mathrm{Y}$ direction, other than model without tower, again model 4 and model 9 expresses the least values.

3. When analyzing shear forces Fx, Fy and Fz when beam parallel to $\mathrm{Z}$ direction for building other than model without tower, the value of Fx, Fy and Fz has seems to be least in model 3 and 8 for Fx and Fz, model 4 and 9 for Fy. When determining moments My and Mz when beam parallel to $\mathrm{Z}$ direction, other than model without tower, again model 4 and 9 expresses the least values for Mz model 3 and 8 for My.

4. Nodal displacement for building seems to be least in model 2 and 7 for $\mathrm{X}$ and $\mathrm{Z}$ direction and for story drift, model 3 and 8 shows least values among all tower placings.

5. Nodal displacement for tower shows the least values for model 4 and 9 for $\mathrm{X}$ direction, since the unit values are very less; model 4 and 9 again shows the least values for $\mathrm{Z}$ direction. Axial forces in compression obtained a least value for model 4 and 9 and the same model shows least values in tension.

6. Hence best suitable location of tower by considering different result parameters seems to be tower at center of short size of the building roof i.e. model 4 for $\mathrm{G}+6$ storey building and model 9 for $\mathrm{G}+10$ storey building.

\section{ACKNOWLEDGEMENT}

We do extremely thankful and respectful to our mentor Prof. GajendraVerma, Assistant Professor, Department ofCivil Engineering, Sri Aurobindo Instiute of Technology, Indore (M. P.); that he always points to critical insights during the entire work, guides us, helps us discover the fun of devising the state of the art solutions.

\section{REFERENCES}

[1]. Malviya Suyash, Jamle Sagar, (2019), , "Determination of optimum location of rooftop telecommunication tower over multistory building under seismic loading”, pp. 65-73.

[2]. Rajoriya Sourabh, Pathak K.K., Vyas Vivekanand (2016), "Analysis of Transmission Tower for Seismic Loading Considering Different Height and Bracing System”, pp. 108-118.

[3]. Shailesh S. Goral, Prof. S. M. Barelikar (2015), "Influence of Structure Characteristics on Earthquake Response Under Different Position of Rooftop Telecommunication Towers", International Journal of Engineering Sciences \& Research Technology, ISSN 2277-9655, Vol. 4, Issue 10, pp. 73-78.

[4]. Soltanzadeh Gholamreza, Shad Hossein, Vafaei Mohammadreza, Adnan Azlan (2014), "Seismic Performance of 4-Legged Self-supporting Telecommunication Towers”, pp. 319-332.

[5]. Shah Hemal J, Dr. Desai Atul K (2014), "Seismic Analysis of Tall TV Tower Considering Different Bracing Systems", pp. 113-119

[6]. Rajasekharan Jithesh, Vijaya S (2014), "Analysis of Telecommunication Tower Subjected to Seismic \& Wind Loading”, pp. 68-79.

[7]. Preeti C. and Mohan K. Jagan (2013), "Analysis of Transmission Towers with Different Configurations", pp. 450-460.

[8 Bhatt ]. Richa,.Pandey A.D, 1 Prakash Vipu (2013), "Influence of modeling in the response of 
steel lattice mobile tower under wind loading”, pp. 137-144.

[9]. Bhosale Nitin, Kumar Prabhat, Pandey A. D. (2012), "Influence of Host Structure Characteristics on Response of Rooftop Telecommunication Towers", pp. 737-748.

[10]..H Siddesha (2010), "Wind Analysis of Microwave Antenna Towers", pp. 574-584.

[11] Veena G1, Sowjanya G.V2, Naveen K3 (2017): Seismic Analysis of Four Legged Telecommunication Towers using Fluid Viscous Dampers. Pp 2343-2349.

[12] Rola Assi and Ghyslaine McClure (2017) A simplified method for seismic analysis of rooftop telecommunication towers 